\title{
Wenn Erfahrung auf die Sprünge hilft
}

\section{Jürg Unger-Köppel}

Dr. med., Mitglied des FMH-Zentralvorstandes, Departementsverantwortlicher Stationäre Versorgung und Tarife

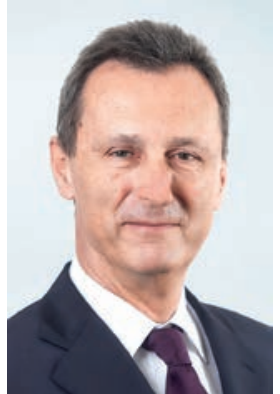

2016 hatte ich als neu gewähltes Mitglied des FMH-Zentralvorstands die Aufgabe, das Departement «Stationäre Versorgung und Tarife» zu übernehmen. In den Tariffragen wurde sehr gute Arbeit geleistet und es war gemeinsam mit der hervorragenden Abteilungsleiterin sorgfältige Kontinuität gefragt. Der Ansatz für Innovation lag eher beim Thema "Stationäre Versorgung». Die Jugend macht die Zukunft des Spitals aus und in vielen Gesprächen mit Studierenden und jungen Ärztinnen und Ärzten hat sich eines vor allem herauskristallisiert: Jene jungen Menschen, die nicht aus einer Arztfamilie stammen, tun sich häufig schwerer mit der Planung ihrer Medizin-Karriere. Sie wissen oft wenig über mögliche Praktika, Weiterbildungsstellen oder gezielte Karriereplanung. Und genau hier kommt nun die ältere Generation zum Zug. Die «Silberrücken», wie die um

Die "Jungen" waren sehr zufrieden und empfanden die Gespräche in den meisten Fällen als sehr hilfreich.

viele Jahre reiche erfahrene Generation liebevoll genannt wird, soll ihr Wissen an die Jungen weitergeben. So ist die Idee von Coach my Career entstanden.

Weil 2016 gleichzeitig auch der Zusammenhalt der FMH im Kontext der Urabstimmung zum neuen ambulanten Tarif TAR... - wie hiess der Tarif damals gerade? - hart auf die Probe gestellt wurde, war es naheliegend, ein gemeinsames Projekt zu starten, das verschiedene grosse Gruppierungen unter dem Dach der FMH an einem Tisch zusammenbrachte. Bei Coach my Career spannten schliesslich SWIMSA, VSAO, VLSS, mfe, SIWF und zwei Departemente der FMH zusammen und erbrachten damit einen weiteren Tatbeweis für die Solidarität über Generationen hinweg und zwischen den Gruppierungen der FMH. Nach intensiven Vorbereitungsarbeiten durch die Projektgruppe ist das Projekt in den Entscheidungsgremien der FMH ausgesprochen wohlwollend aufgenommen worden. Im September 2018 erfolgte der Startschuss mit den ersten Beratungsgesprächen im Rahmen von Coach my Career. Jeweils zwei Coaches stellten sich zusammen einer jungen, ratsuchenden Person zur Verfügung. Schon die ersten Gespräche ver- liefen erfolgreich, wie es in den regelmässigen Feedbacks der Mentees an den VLSS hiess. Die «Jungen» waren sehr zufrieden und empfanden die Gespräche in den meisten Fällen als sehr hilfreich (lesen Sie dazu mehr auf Seite 1412 in dieser Ausgabe)

Auch die Bilanz der Coaches fällt jetzt, nach einem Jahr Arbeit mit den Mentees, durchwegs positiv aus. Bereits arbeiten alle beteiligten Seiten mit viel Enthusiasmus an der Fortsetzung des Projekts. Es ist der gemeinsame Wunsch, sich nicht auf dem bisher Geleisteten auszuruhen, sondern auch bei Coach my Career die Qualität der Arbeit der Ärztinnen und Ärzte zu verbessern. Deshalb wurden verschiedene wichtige Vorschläge zur Optimierung des Projekts erarbeitet, um die Bedürfnisse der Mentees noch besser zu verstehen und gezielter zu bedienen. Gleichzeitig werden die jährlichen Veranstaltungen der SWIMSA und des VSAO an den medizinischen Fakultäten weitergeführt, an denen wichtige Tipps für den Berufseinstieg vermittelt werden. Diese Veranstaltungen gegen Ende des Studiums wurden bereits im ersten Projektjahr von den Studentinnen und Studenten sehr geschätzt.

Wie so oft bei Projekten sind auch bei Coach my Career die Ressourcen ein Thema. Dank dem unermüdlichen Einsatz aller Beteiligten und mit nur wenigen Projektsitzungen und minimalstem finanziellem Aufwand ist Coach my Career zum Fliegen gebracht worden. Dafür gilt es, allen einen grossen Dank auszusprechen.

Und zum Schluss ein ganz privater Gedanke: Wenn der eigene Rücken langsam ergraut, ist man selbst auf dem der Weg zum «Silberrücken». Dies gilt natürlich auch

Es kommen tolle, sehr engagierte und äusserst professionelle junge Ärztinnen und Ärzte nach.

für diejenigen Ärztinnen und Ärzte, die sich bis anhin um einen gekümmert haben und denen man die eigenen medizinischen Anliegen anvertraut hat. Auch sie werden schon bald in den wohlverdienten Ruhestand gehen. Spätestens dann fragt man sich, wer sich künftig um einen sorgen wird. Auf diese Frage gibt es eine erfreuliche Antwort: Es kommen tolle, sehr engagierte und äusserst professionelle junge Ärztinnen und Ärzte nach. 